\title{
Local Application of Strontium in a Calcium Phosphate Cement System Accelerates Healing of Soft Tissue Tendon Grafts in Anterior Cruciate Ligament Reconstruction
}

\section{Experiment Using a Rabbit Model}

\author{
Guan-Ming Kuang, ${ }^{*}$ W.P. Yau, ${ }^{\dagger \neq}$ William W. Lu, ${ }^{\ddagger}$ and K.Y. Chiu ${ }^{\ddagger}[A Q:$, \\ Investigation performed at the University of Hong Kong, Hong Kong, China
}

\begin{abstract}
Background: Healing of soft tissue tendon grafts within the bone tunnel in anterior cruciate ligament (ACL) reconstruction is known to be slower than that of bone-patellar tendon-bone grafts. There are attempts to accelerate healing of the graft within the bone tunnel. One of the methods is the use of strontium-enriched calcium phosphate cement (Sr-CPC). Early results in animal studies have been encouraging, although it is not known whether the accelerated healing was solely caused by the effect of strontium within the cement or by the calcium phosphate cement (CPC) itself.
\end{abstract}

Hypothesis: There would be differences between Sr-CPC and conventional CPC in terms of the effect on healing of soft tissue tendon grafts within the bone tunnels in ACL reconstruction.

Study Design: Controlled laboratory study.

Methods: A total of 30 single-bundle ACL reconstruction procedures were performed in 15 rabbits with the use of an Achilles tendon allograft. The graft on the left limb was coated with Sr-CPC, while that on the right limb was coated with CPC. Three animals each were sacrificed for histological and histomorphometric analyses at 3, 6, 9, 12, and 24 weeks after surgery.

Results: In the Sr-CPC group, early formation of Sharpey fibers was present at 6 weeks after surgery, while early remodeling of a graft-fibrocartilage-bone junction was noted at 12 weeks. In the CPC group, early formation of Sharpey fibers was only found at 9 to 12 weeks after surgery. At 24 weeks, a direct enthesis was found in both groups. According to the histomorphometric score, graft healing in the Sr-CPC group took place 3 weeks faster than that in the CPC group at and before 12 weeks; however, there was no difference between the groups at 24 weeks.

Conclusion: The local application of strontium in a CPC system leads to accelerated graft healing within the bone tunnels.

Clinical Relevance: The use of Sr-CPC to enhance graft-bone healing may improve the clinical results of ACL reconstruction using soft tissue tendon grafts.

Keywords: anterior cruciate ligament (ACL); strontium; calcium phosphate cement (CPC); tendon-bone healing

\footnotetext{
${ }^{\dagger}$ Address correspondence to W.P. Yau, Department of Orthopaedics and Traumatoloav L KaShing Faculty of Medicine, University of Hong Kong, [AQ: 2], , fong SAR, China (e-mail: peterwpy@hkucc.hku.hk). *Shenzhen boratory for Innovative Technology in Orthopaedic Trauma, Department of Orthopaedics and Traumatalogy, Shenzhen Hospital, University of Hong Kong, Shenzhen, China

${ }^{\ddagger}$ Department of Orthopaedics and Traumatol 2 KaShing Faculty of Medicine, University of Hong Kong, Hong Kong, Cnina.

The authors declared that they have no conflicts of interest in the authorship and publication of this contribution.
}

The American Journal of Sports Medicine, Vol. XX, No. X DOI: $10.1177 / 0363546514549536$

(c) 2014 The Author(s)
Surgery with a soft tissue tendon graft (eg, hamstring graft) is one of the most common procedures in anterior cruciate ligament (ACL) reconstruction. ${ }^{2,14,17}$ The success rate is typically greater than $90 \%$, and donor site morbidities are significantly less than those of bone-patellar tendon-bone grafts. ${ }^{3,8,10}$ However, the long healing time of soft tissue tendon grafts within the bone tunnels is still a concern for most surgeons. Animal studies have shown that the grafted tendon attaches to the bone tunnels by means of Sharpey fibers starting at approximately 12 weeks after surgery. ${ }^{4,9,24,25}$ It is a common practice for functional drills to be started at 3 to 6 months after surgery and for the patient to be advised to return to play 6 to 9 months after the index operation. ${ }^{6}$ 


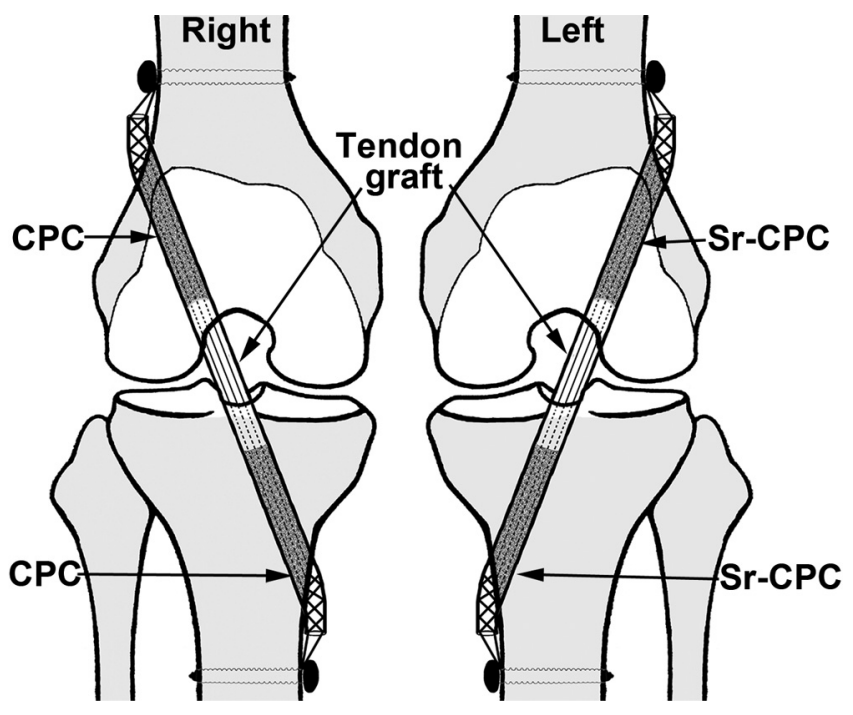

Figure 1. Surgical design. The graft on the left limb was coated with strontium-enriched calcium phosphate cement (Sr-CPC). The graft on the right limb was coated with conventional calcium phosphate cement (CPC). The area of the cement coating on the tendon allograft surface is marked as gray, while the uncoated area of the allograft is marked as white.

In recent years, methods have been reported that enhance healing of soft tissue tendon grafts within the bone tunnels in animal models of ACL reconstruction..$^{9,12,18,22,24-26,28}$ It has been shown that graft healing within the bone tunnels can be enhanced by a local application of calcium phosphate cement (CPC), which is a resorbable and osteoconductive biomaterial. ${ }^{9,18,24,25}$ Recently, it was shown that the osteoconductivity of CPC can be enhanced by the incorporation of strontium. ${ }^{11,19}$ In a recent study using strontium-enriched calcium phosphate cement (Sr-CPC) in a rabbit model of ACL reconstruction, it was shown that a coating of the Achilles tendon allograft with Sr-CPC, with the molar ratio for $\mathrm{Sr} /(\mathrm{Sr}+\mathrm{Ca})$ being $10 \%$, led to an acceleration of graft healing within the bone tunnels as compared with the untreated control group. ${ }^{13}$ However, it was not known whether the accelerated healing was solely caused by the effect of strontium within the cement or by CPC itself. To answer this question, the previous experiment was repeated comparing a graft treated with Sr-CPC with a CPC-treated graft in a rabbit model of ACL reconstruction. It was hypothesized that there would be differences between the Sr-CPC group and CPC group in terms of the effect on healing of soft tissue tendon grafts within the bone tunnels.

\section{MATERIALS AND METHODS}

A total of 30 ACL reconstruction procedures using an Achilles tendon allograft were performed in fifteen 5-month-old New Zealand White rabbits with a mean
TABLE 1

Composition of Sr-CPC and $\mathrm{CPC}^{\alpha}$

\begin{tabular}{lll}
\hline & \multicolumn{1}{c}{ Sr-CPC } & CPC \\
\hline $\begin{array}{l}\text { Sr/(Sr }+ \text { Ca }) \\
\text { molar ratio }\end{array}$ & $10 \%$ & $0 \%$ \\
Powder phase & TTCP + DCPA & TTCP + DCPA \\
& + DSPA & \\
Liquid phase & $20 \mathrm{wt} \%$ citric acid & $20 \mathrm{wt} \%$ citric acid \\
& $+12 \mathrm{wt} \% \mathrm{PVP}$ & $+12 \mathrm{wt} \% \mathrm{PVP}$ \\
Liquid/powder ratio & $0.5 \mathrm{~mL} / \mathrm{g}$ & $0.5 \mathrm{~mL} / \mathrm{g}$ \\
\hline
\end{tabular}

${ }^{a} \mathrm{Ca}$, calcium; CPC, calcium phosphate cement; DCPA, dicalcium phosphate anhydrous; DSPA, strontium hydrogen phosphate; PVP, polyvinylpyrrolidone K-30; Sr, strontium; Sr-CPC, strontium-enriched calcium phosphate cement; TTCP, tetracalcium phosphate; wt \%, percentage by weight.

weight of $2.8 \mathrm{~kg}$ (range, 2.5-3.0 kg). The graft on the left limb was coated with Sr-CPC, while that on the right limb was coated with CPC (Figure 1). The experiment was conducted with a protocol approved by the Committee on the Use of Live Animals in Teaching and Research of our institute.

The Sr-CPC was prepared according to a reported protocol (Table 1). ${ }^{13}$ The powder phase of Sr-CPC consisted of tetracalcium phosphate (TTCP; Wako), dicalcium phosphate anhydrous (DCPA; Alfa Aesar), and strontium hydrogen phosphate (DSPA; Sigma-Aldrich). The total molar ratio for $\mathrm{Sr} /(\mathrm{Sr}+\mathrm{Ca})$ was $10 \%$. The liquid phase contained 20 percentages by weight (wt\%) of citric acid (Wako) and 12 wt\% polyvinylpyrrolidone K-30 (PVP; Wako). The cement paste was then prepared by mixing the liquid and powder phases in a ratio of $0.5 \mathrm{~mL} / \mathrm{g}$. For CPC, the powder phase was free of strontium, consisting of an equimolar ratio of TTCP and DCPA. The liquid phase and the liquid/powder ratio of CPC for mixing were the same as those of Sr-CPC (Table 1).

One-stage sequential bilateral ACL reconstruction was carried out in rabbits according to a published protocol. ${ }^{13}$ Arthrotomy was performed, and the native ACL was cut. Bone tunnels ( $2 \mathrm{~mm}$ in diameter, $10 \mathrm{~mm}$ in length) were prepared in both the femur and tibia at the footprints of the native ACL. Fresh-frozen rabbit Achilles tendon allografts with a diameter of $2 \mathrm{~mm}$ were used. Both ends of the tendon graft were precoated with the cement before it was inserted into the bone tunnels. In addition, the cement was injected into the bone tunnels from the far cortex exits of the bone tunnels before transfer of the graft into the bone tunnels. The graft was inserted using an inside-out approach to minimize the deposition of cement inside the joint. Any residual cement in the joint and around the intra-articular exits of the bone tunnels was removed. The graft was then fixed on the femur and tibia using a tied-over-post technique on a $2.7 \mathrm{~mm}$-diameter stainless steel cortical screw with a washer. Surgery was performed on the contralateral knee with the graft treated with CPC alone. Three animals were sacrificed in each group at 3, 6, 9,12 , and 24 weeks after surgery. 
TABLE 2

Results of Histomorphometric Analysis ${ }^{a}$

\begin{tabular}{lccc}
\hline & Sr-CPC Group & CPC Group & $P$ Value $^{b}$ \\
\hline $3 \mathrm{wk}$ & $1.9 \pm 0.4$ & $1.2 \pm 0.4$ & $<.001$ \\
$6 \mathrm{wk}$ & $3.3 \pm 0.4$ & $2.0 \pm 0.4$ & $<.001$ \\
$9 \mathrm{wk}$ & $4.6 \pm 0.3$ & $2.7 \pm 0.6$ & $<.001$ \\
$12 \mathrm{wk}$ & $6.6 \pm 0.2$ & $4.1 \pm 0.6$ & $<.001$ \\
$24 \mathrm{wk}$ & $6.8 \pm 0.2$ & $6.7 \pm 0.2$ & .82 \\
\hline
\end{tabular}

${ }^{a}$ Values are expressed as mean $\pm \mathrm{SD}$. CPC, calcium phosphate cement; Sr-CPC, strontium-enriched calcium phosphate cement.

${ }^{b}$ Mann-Whitney $U$ test.

The specimens were fixed in neutralized formalin solution, followed by immersion in $10 \%$ formic acid for decalcification. The decalcified specimens were then dehydrated and embedded in paraffin, followed by sectioning at $6-\mu \mathrm{m}$ thickness in an orientation parallel to the long axis of the bone tunnels. The sections were stained with hematoxylin and eosin and safranin $\mathrm{O}$.

Histomorphometric analysis was performed by the first author [AQ: 3] a ng to a protocol in the published literature. $^{13,27}$ The hiner was blinded to the group when conducting this analysis. Healing of the graft within the bone tunnel was graded by 3 criteria: new bone formation, graft binding to the adjacent tissue, and fibrocartilage formation according to a score of 0 to 3 for each criterion and in total 0 to 9 for a slide. For each histological section, the whole graft-bone interface was divided into 6 zones, of which 2 zones represented the proximal one third of the bone tunnel to the articular surface, the other 2 zones represented the middle third of the bone tunnel, and the remaining 2 zones represented the distal one third of the bone tunnel to the articular surface. Each zone was independently analyzed, and the healing score of each zone was obtained. The mean score of these 6 zones was calculated to represent the overall score of that histological section. Seven slides were randomly selected from each specimen (tibia and femur), resulting in a total of 28 histomorphometric scores for each animal (14 from the Sr-CPC-treated limb and 14 from the CPC-treated limb). A nonparametric test (Mann-Whitney $U$ test) was used to calculate the statistical difference between the histomorphometric score of the Sr-CPC- and CPC-treated limbs at different time points (SPSS v12.0J for Windows, SPSS Inc). Statistical significance was assumed if the $P$ value was $<.05$.

\section{RESULTS}

The results of the histomorphometric analysis are shown in Table 2. A higher score was found in the Sr-CPC group as compared with the CPC group at 3, 6, 9, and 12 weeks after surgery (Mann-Whitney $U$ test) (Table 2). It was observed that graft healing in the Sr-CPC-treated limb was in general 3 weeks faster than that in the CPC group at or before 12 weeks after the index operation. No difference was found in the score between the Sr-CPC and CPC groups at 24 weeks after surgery (Table 2).
At 3 weeks after surgery, in the CPC group, most parts of the graft-bone interface were filled with cement (Figure 2A). However, in the Sr-CPC group, the resorption of Sr-CPC was evident, with the newly formed bone surrounding the residual cement in the graft-bone interface (Figure 2B).

At 6 weeks, close apposition of new woven bone to the peripheral portion of the tendon graft was noted in some parts of the graft-bone interface in the CPC group (Figure 2C). However, for the Sr-CPC group, the cement had resorbed. The gap between the graft and bone had been filled by newly formed bone. The formation of fibrous connective tissue resembling Sharpey fibers was also observable in parts of the graft-bone interface of the Sr-CPC group (Figure 2D).

At 9 weeks, there was evidence of early formation of Sharpey-like fibers in a few portions of the graft-bone interface in the CPC group (Figure 2E). On the other hand, in the Sr-CPC group, Sharpey fibers had formed throughout most of the graft-bone interface (Figure 2F).

At 12 weeks, in the CPC group, an indirect type of healing (Sharpey fiber formation) was observable in the graftbone interface (Figure 3A and 3C). In the Sr-CPC group, early remodeling with a graft-fibrocartilage-bone transition zone was noted (Figure 3B, 3D, and 3E).

At 24 weeks, healing of the tendon graft within the bone tunnel with the presence of a direct type of enthesis (graftfibrocartilage-bone transition) was noted in both the Sr-CPC and CPC groups (Figure 4).

\section{DISCUSSION}

This study showed that Sr-CPC accelerated healing of Achilles tendon allografts within the bone tunnels in comparison with a CPC-treated graft in a rabbit model of ACL reconstruction at $3,6,9$, and 12 weeks after the index operation. At 24 weeks after surgery, graft healing in the Sr-CPC and CPC groups was comparable, with a directtype ligament-bone attachment being observed in both groups.

The finding of the CPC group in the current study was comparable with those in the published literature. ${ }^{24,25}$ In rabbit models of ACL reconstruction using calcium phosphate-treated autografts, new bone formation was observed at the graft-bone interface 6 weeks after surgery. The formation of Sharpey fibers was observed 12 weeks after surgery. ${ }^{24,25}$ In this study, new bone formation was observed at 6 weeks after surgery in the CPC group; early formation of Sharpey fibers was observed at 9 weeks in portions of the graft-bone interface.

In concurrence with a previously published article, ${ }^{13}$ the current study demonstrated that coating the tendon graft with Sr-CPC resulted in enhanced new bone formation at 3 weeks, indirect healing at 6 weeks, and remodeling into a direct-type enthesis at 12 weeks.

It has been reported that strontium showed anabolic and anticatabolic effects on the skeleton system. In cell experiments, it was shown that strontium increased the expression of the osteoblast markers alkaline phosphatase, 


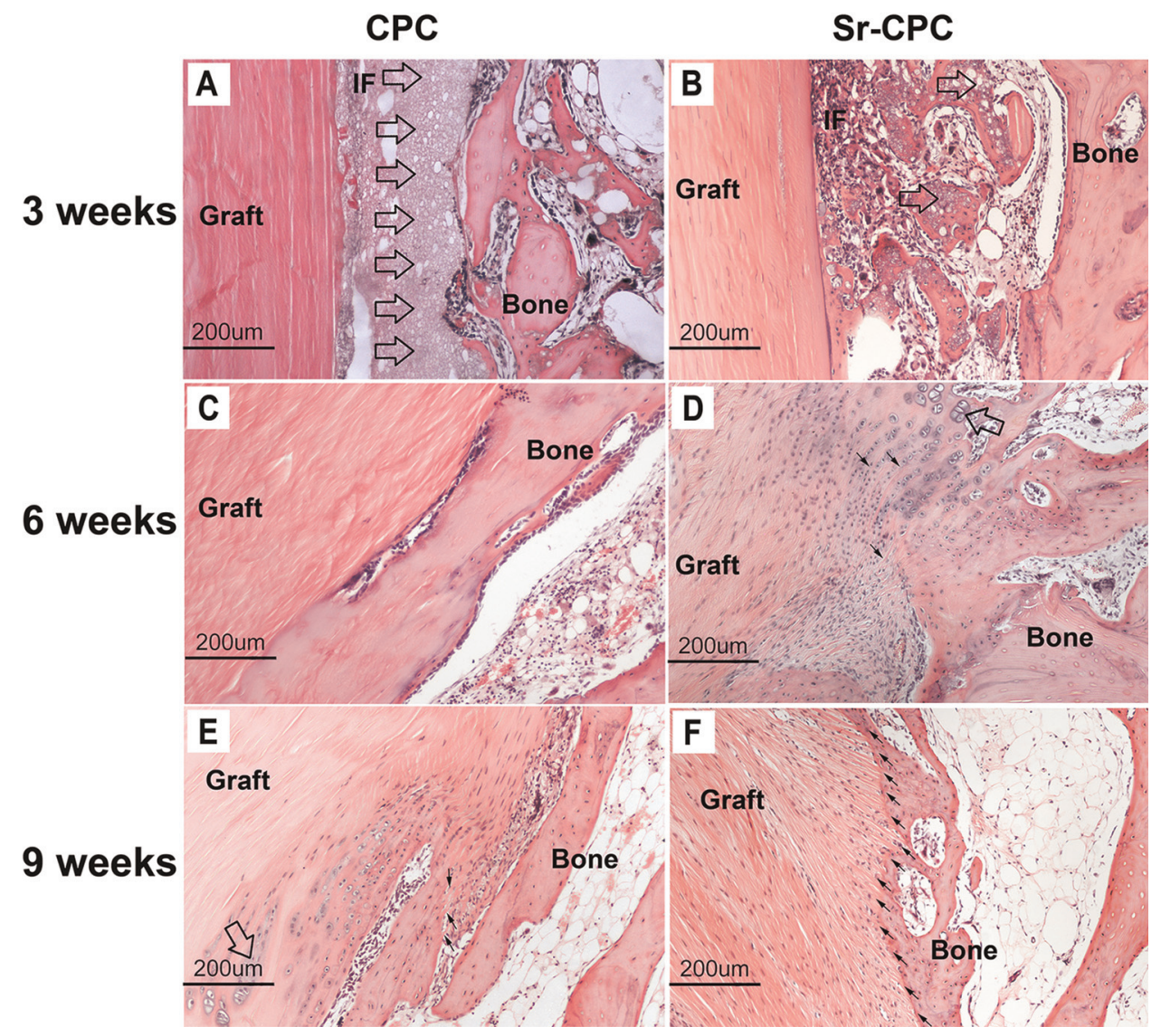

Figure 2. Representative images of the graft-bone interface (IF) at (A, B) 3 weeks, (C, D) 6 weeks, and (E, F) 9 weeks after surgery. (A, B) The IF was filled with fibrovascular tissue and residual cement (clear arrows). (B) New bone was found surrounding the residual cement in the IF in the strontium-enriched calcium phosphate cement (Sr-CPC) group (clear arrows). (C) Close apposition of woven bone was observed in the calcium phosphate cement (CPC)-treated limbs at 6 weeks. (D) The early formation of Sharpey fibers (solid arrows) and a cluster of chondrocyte-like cells (clear arrow) were observed in the Sr-CPC-treated limbs at 6 weeks. (E) The formation of Sharpey-like fibers and chondrocyte-like cells (clear arrow) were noted in a few portions of the IF in the CPC group at 9 weeks [AQ: 4]. 7 arpey fibers (black arrows) were found in the majority of the tendon-bone interface, perpendicularly connecting the graft 1 bone tunnel wall. Hematoxylin and eosin staining $(\times 100)$.

bone sialoprotein, and osteocalcin as well as bone nodule numbers in bone marrow stromal cells ${ }^{5,7}$ and osteoblasts, ${ }^{29}$ showing an anabolic effect on bone remodeling. On the other hand, strontium inhibited the expression of carbonic anhydrase II and vitronectin receptor in stimulated osteoclasts, indicating an inhibition of the osteoclast differentiation and a reduction in osteoclast resorbing activity ${ }^{1,5,23}$ and showing an anticatabolic effect on bone remodeling. In animal studies, it was shown that strontium exerted a bone-forming effect on normal and osteoporotic rat trabecular bone, ${ }^{20}$ stimulated new bone formation in an ovariectomized goat model, ${ }^{15}$ and prevented bone loss by depressing bone resorption without reducing bone formation in rats. ${ }^{16}$ Therefore, the anabolic and anticatabolic effects of strontium may be some of the reasons leading to enhanced new bone formation at the graft-bone interface of the Sr-CPC group at 3 weeks after surgery.

In this study, although earlier formation of Sharpey fibers was observed at 6 weeks after surgery in the
Sr-CPC group as compared with the CPC group, the exact mechanism leading to this outcome is still unknown from the data of the current study. Sharpey fibers are thought to be formed by the progressive mineralization of collagen fibers of the ligaments or the periosteum by advancing bone during growth. ${ }^{21}$ It is not known whether the earlier formation of Sharpey fibers observed in the Sr-CPC group in this study was associated with enhanced new bone formation in the graft-bone interface at an earlier healing stage or if this outcome was because of a direct effect exerted by strontium in the CPC system [AQ: 6]

In this study, early remodeling of the graft-fibro 2 gebone junction was noted to start at 12 weeks in the far end of the bone tunnel, near the site of initial fixation with the tie-over-post technique. However, at 24 weeks, the graftfibrocartilage transitional zone was observed at the intraarticular exit site of the graft. This simulated the native ACL-ligament-bone [AQ: 7] mon. However, there was no histological study perforn tween 12 and 24 weeks 


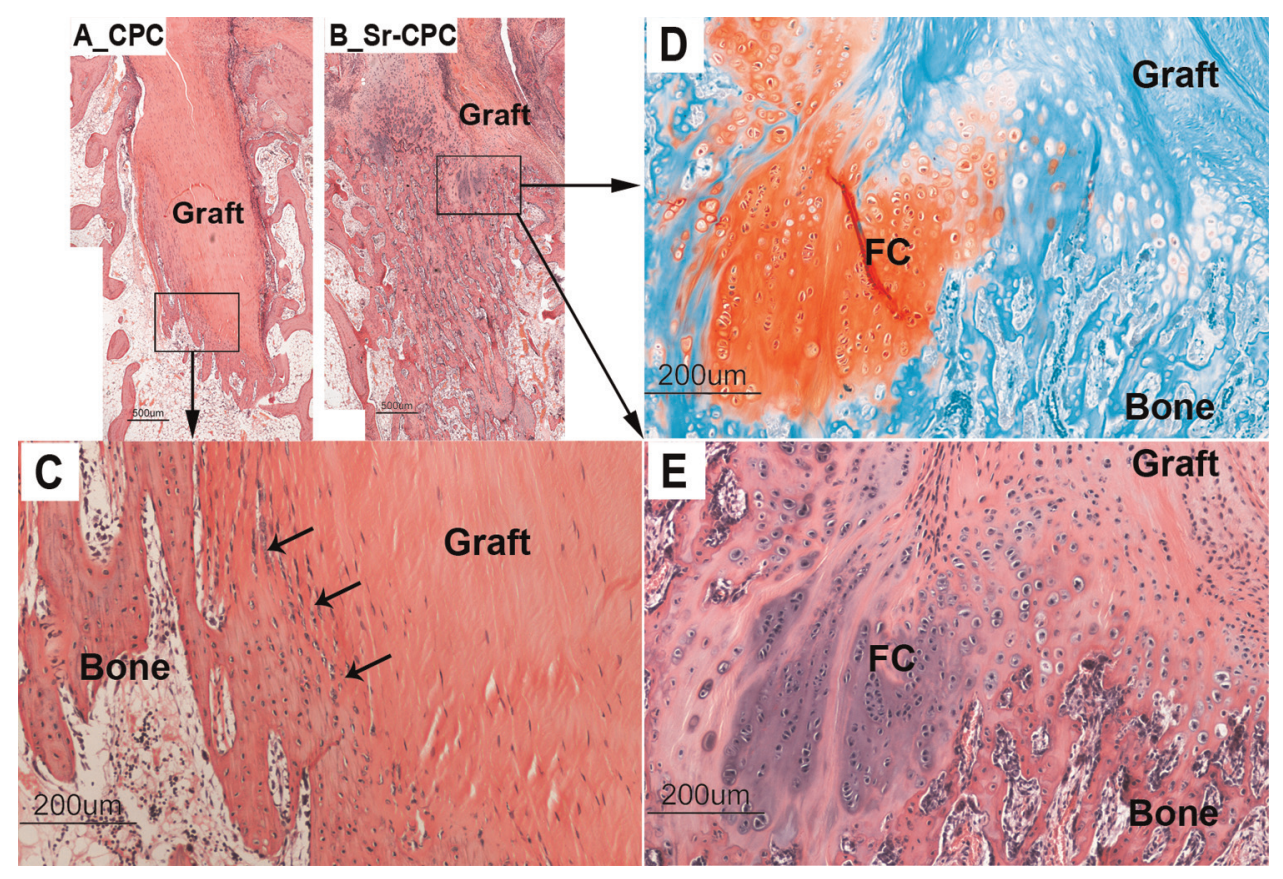

Figure 3. Representative images of the graft-bone interface at 12 weeks after surgery. (A) Ultra low-power image of the graftbone component of a calcium phosphate cement (CPC)-treated limb. Hematoxylin and eosin (H\&E) staining. (B) Ultra low-power image of the graft-bone component of a strontium-enriched calcium phosphate cement (Sr-CPC)-treated limb. H\&E staining. (C) High-power image of the rectangular area in A. H\&E staining (×100). (D) High-power image of the rectangular area in B. Safranin O staining (×100). (E) High-power image of the rectangular area in B. H\&E staining (×100). Arrows indicate Sharpey fibers. FC, fibrocartilage.

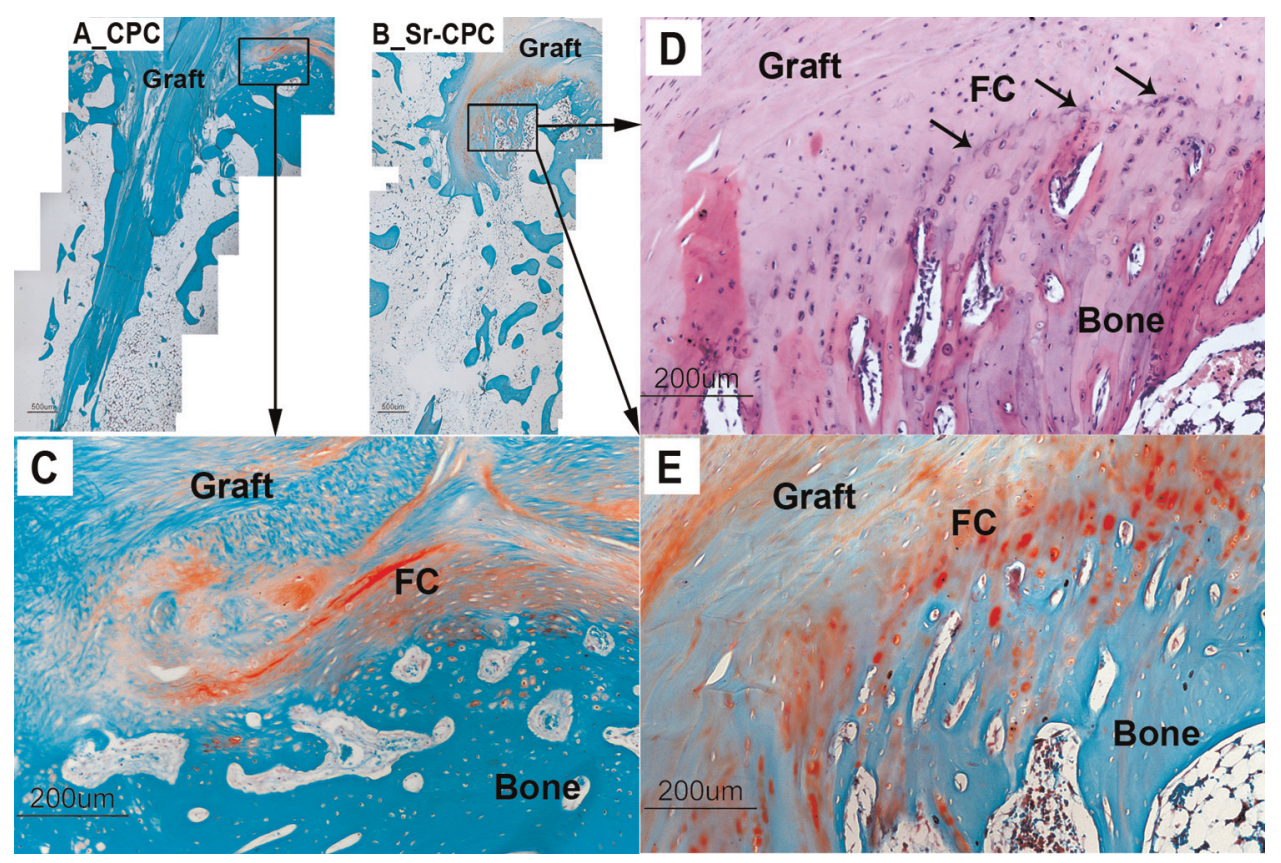

Figure 4. Representative images of the graft-bone interface at 24 weeks after surgery. (A) Ultra low-power image of the graftbone component of a calcium phosphate cement (CPC)-treated limb. Safranin O staining. (B) Ultra low-power image of the graft-bone component of a strontium-enriched calcium phosphate cement (Sr-CPC)-treated limb. Safranin $\mathrm{O}$ staining. (C) High-power image of the rectangular area in A. Safranin O staining $(\times 100)$. (D) High-power image of the rectangular area in B. Hematoxylin and eosin staining $(\times 100)$. (E) High-power image of the rectangular area in B. Safranin $O$ staining $(\times 100)$. Arrows in $\mathrm{D}$ indicate the mineralization line. FC, fibrocartilage. Arrows in $\mathrm{D}$, cement line [AQ: 5$]$

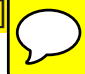


after the index operation; thus, it is not known what happened with the healing process during this period. Further studies are needed to clarify this.

There are several limitations to this study. First, in total, 15 rabbits were used, and the sample size was relatively small. However, the utilization of 1-stage sequential bilateral ACL reconstruction made a direct comparison possible between the Sr-CPC group and CPC group in a specific animal. Furthermore, a histomorphometric examination provided additional evidence to allow a quantified comparison of the groups.

Second, biomechanical properties of the graft-bone component were not studied. We did not know whether the improved and accelerated histological maturity of the graft-bone interface in the $\mathrm{Sr}-\mathrm{CPC}$ group at 3 to 12 weeks led to better biomechanical outcomes as compared with the CPC group. In addition, although a direct type of insertion was found at 24 weeks after surgery, it was not made clear whether this resembled the normal biomechanical properties of a native ACL's direct enthesis [AQ: 8]

Third, the cellular mechanism by whic 2 ontium accelerates graft healing within the bone tunnel is still unknown. It was observed that there is accelerated new bone formation within the gap between the graft and the bone tunnel, and it was also observed that there was an earlier appearance of indirect healing, which is characterized by the formation of Sharpey fibers at the graft-bone interface in the Sr-CPC group as compared with the CPC group. The local application of strontium may lead to enhanced new bone formation in the initial gap between the graft and the bone tunnel because of its dual anabolic and anticatabolic effects on bone remodeling. However, it is not known whether strontium exerts a direct effect on the formation of Sharpey fibers, leading to an earlier formation of Sharpey fibers in the Sr-CPC group at 6 weeks after surgery, or the earlier formation of Sharpey fibers is associated with enhanced new bone formation at an early healing stage [AQ: 9].

Fourth, although the cu 2 study provides evidence for accelerated healing of the graft within the bone tunnel using Sr-CPC, the histological change that occurred in the intra-articular portion of the graft was not studied. Thus, it is not known at this juncture whether it will be accompanied by an acceleration of ligamentization of the midsubstance of the intra-articular portion of the graft. Further studies are needed to investigate the histological changes in the intra-articular portion of the graft after the treatment of Sr-CPC.

Fifth, despite the current study's focus on biological healing of the graft within the bone tunnel, one should be aware that there are many possible reasons for the observed failure of ACL reconstruction in clinical practice. These include incorrect tunnel positioning, suboptimal hardware fixation, technical errors during surgery, graft impingement, concomitant collateral ligament laxity, premature return to sport, repeated trauma, and problems in graft ligamentization.

Finally, although this study showed that the local application of strontium in a CPC system accelerated healing of soft tissue tendon grafts within the bone tunnels in an animal model of ACL reconstruction, whether the results of this basic science study can be extrapolated to clinical practice is unanswered at the present moment. The overall anabolic and anticatabolic effects of strontium need to be further evaluated in clinical models. A randomized controlled trial comparing ACL reconstruction using Sr-CPC-coated soft tissue tendon grafts (eg, quadrupled hamstring grafts) and conventional ACL reconstruction using soft tissue tendon grafts in collegiate or professional players will help to provide answers to this question. As the CPC system is injectable, the clinical application of strontium to the graft-bone interface can be achieved by local injections of Sr-CPC through specific devices. This is technically possible in arthroscopic assisted ACL reconstruction in clinical practice. The overall anabolic and anticatabolic effects of strontium on the bone tunnel can be assessed by quantitative computed tomography performed at 3 and 6 months after the index operation. Healing of the graft within the bone tunnel and ligamentization of the intra-articular portion of the graft can be assessed by interval magnetic resonance imaging performed at 3 months, 6 months, 9 months, and 1 year to provide data on the overall recovery time.

\section{CONCLUSION}

In the present study, healing of the Sr-CPC-treated soft tissue tendon allografts within the bone tunnel in a rabbit model of ACL reconstruction was found to be faster than that in the CPC-treated grafts up to 12 weeks after surgery.

\section{REFERENCES}

1. Baron R, Tsouderos Y. In vitro effects of S12911-2 on osteoclast function and bone marrow macrophage differentiation. Eur J Pharmacol. 2002;450(1):11-17.

2. Bartlett RJ, Clatworthy MG, Nguyen TN. Graft selection in reconstruction of the anterior cruciate ligament. J Bone Joint Surg Br. 2001;83(5):625-634.

3. Biau DJ, Katsahian S, Nizard R. Hamstring tendon autograft better than bone-patellar tendon-bone autograft in $\mathrm{ACL}$ reconstruction: a cumulative meta-analysis and clinically relevant sensitivity analysis applied to a previously published analysis. Acta Orthop. 2007;78(5): 705-707, author reply 707-708.

4. Blickenstaff KR, Grana WA, Egle D. Analysis of a semitendinosus autograft in a rabbit model. Am J Sports Med. 1997;25(4):554-559.

5. Bonnelye E, Chabadel A, Saltel F, Jurdic P. Dual effect of strontium ranelate: stimulation of osteoblast differentiation and inhibition of osteoclast formation and resorption in vitro. Bone. 2008;42(1):129138.

6. Cascio BM, Culp L, Cosgarea AJ. Return to play after anterior cruciate ligament reconstruction. Clin Sports Med. 2004;23(3):395-408, ix.

7. Choudhary S, Halbout P, Alander C, Raisz L, Pilbeam C. Strontium ranelate promotes osteoblastic differentiation and mineralization of murine bone marrow stromal cells: involvement of prostaglandins. J Bone Miner Res. 2007;22(7):1002-1010.

8. Ejerhed L, Kartus J, Sernert N, Kohler K, Karlsson J. Patellar tendon or semitendinosus tendon autografts for anterior cruciate ligament reconstruction? A prospective randomized study with a two-year follow-up. Am J Sports Med. 2003;31(1):19-25. 
9. Huangfu X, Zhao J. Tendon-bone healing enhancement using injectable tricalcium phosphate in a dog anterior cruciate ligament reconstruction model. Arthroscopy. 2007;23(5):455-462.

10. Krych AJ, Jackson JD, Hoskin TL, Dahm DL. A meta-analysis of patellar tendon autograft versus patellar tendon allograft in anterior cruciate ligament reconstruction. Arthroscopy. 2008;24(3):292-298.

11. Kuang GM, Yau WP, Lam WM, et al. An effective approach by a chelate reaction in optimizing the setting process of strontiumincorporated calcium phosphate bone cement. J Biomed Mater Res B Appl Biomater. 2012;100(3):778-787.

12. Kuang GM, Yau WP, Lu WW, Chiu KY. Osteointegration of soft tissue grafts within the bone tunnels in anterior cruciate ligament reconstruction can be enhanced. Knee Surg Sports Traumatol Arthrosc. 2010;18(8):1038-1051.

13. Kuang GM, Yau WP, Lu WW, Chiu KY. Use of a strontium-enriched calcium phosphate cement in accelerating the healing of soft-tissue tendon graft within the bone tunnel in a rabbit model of anterior cruciate ligament reconstruction. Bone Joint J. 2013;95(7):923-928.

14. Lawhorn KW, Howell SM. Principles for using hamstring tendons for anterior cruciate ligament reconstruction. Clin Sports Med. 2007; 26(4):567-585.

15. Li Z, Lu WW, Chiu PK, et al. Strontium-calcium coadministration stimulates bone matrix osteogenic factor expression and new bone formation in a large animal model. J Orthop Res. 2009;27(6):758-762.

16. Marie PJ, Hott M, Modrowski D, et al. An uncoupling agent containing strontium prevents bone loss by depressing bone resorption and maintaining bone formation in estrogen-deficient rats. J Bone Miner Res. 1993;8(5):607-615.

17. Nakase J, Kitaoka K, Matsumoto K, Tomita K. Facilitated tendonbone healing by local delivery of recombinant hepatocyte growth factor in rabbits. Arthroscopy. 2010;26(1):84-90.

18. Pan W, Wei Y, Zhou L, Li D. Comparative in vivo study of injectable biomaterials combined with BMP for enhancing tendon graft osteointegration for anterior cruciate ligament reconstruction. J Orthop Res. 2011;29(7):1015-1021.

19. Panzavolta S, Torricelli P, Sturba L, Bracci B, Giardino R, Bigi A. Setting properties and in vitro bioactivity of strontium-enriched gelatin-calcium phosphate bone cements. J Biomed Mater Res A. 2008;84(4):965-972.

20. Peng S, Liu XS, Wang T, et al. In vivo anabolic effect of strontium on trabecular bone was associated with increased osteoblastogenesis of bone marrow stromal cells. J Orthop Res. 2010;28(9):12081214.

21. Rodeo SA, Arnoczky SP, Torzilli PA, Hidaka C, Warren RF. Tendonhealing in a bone tunnel: a biomechanical and histological study in the dog. J Bone Joint Surg Am. 1993;75(12):1795-1803.

22. Soon MY, Hassan A, Hui JH, Goh JC, Lee EH. An analysis of soft tissue allograft anterior cruciate ligament reconstruction in a rabbit model: a short-term study of the use of mesenchymal stem cells to enhance tendon osteointegration. Am J Sports Med. 2007;35(6): 962-971.

23. Takahashi N, Sasaki T, Tsouderos Y, Suda T. S 12911-2 inhibits osteoclastic bone resorption in vitro. J Bone Miner Res. 2003;18(6):10821087.

24. Tien YC, Chin TT, Lin JH, Ju CP, Lin SD. Augmentation of tendonbone healing by the use of calcium-phosphate cement. J Bone Joint Surg Br. 2004;86(7):1072-1076.

25. Wen CY, Qin L, Lee KM, Chan KM. The use of brushite calcium phosphate cement for enhancement of bone-tendon integration in an anterior cruciate ligament reconstruction rabbit model. J Biomed Mater Res B Appl Biomater. 2009;89(2):466-474.

26. Yabroudi MA, Irrgang JJ. Rehabilitation and return to play after anatomic anterior cruciate ligament reconstruction. Clin Sports Med. 2013;32(1):165-175.

27. Yeh W-L, Lin S-S, Yuan L-J, Lee K-F, Lee MY, Ueng SWN. Effects of hyperbaric oxygen treatment on tendon graft and tendon-bone integration in bone tunnel: biochemical and histological analysis in rabbits. J Orthop Res. 2007;25(5):636-645.

28. Youn I, Jones DG, Andrews PJ, Cook MP, Suh JK. Periosteal augmentation of a tendon graft improves tendon healing in the bone tunnel. Clin Orthop Relat Res. 2004;419:223-231.

29. Zhu LL, Zaidi S, Peng Y, et al. Induction of a program gene expression during osteoblast differentiation with strontium ranelate. Biochem Biophys Res Commun. 2007;355(2):307-311. 